\title{
Experimental Challenge to Heavy Element Synthesis under Explosive Burning on Neutron Stars
}

\author{
Shigeru Kubono ${ }^{1,2 *}$ \\ ${ }^{1}$ RIKEN Nishina Center, 2-1 Hirosawa, Wako, Saitama, 351-0198 Japan \\ ${ }^{2}$ Center for Nuclear Study, the University of Tokyo, 2-1 Hirosawa, Wako, Saitama, 351-0198 Japan
}

\begin{abstract}
Binary stellar systems that involve a neutron star or two neutron stars make interesting phenomena, X-ray bursts and kilo-novae, respectively, which involve explosive burning either in the proton-rich environment or in the neutron-rich environment. These are very important problems for nuclear astrophysics. First, the current effort for the explosive hydrogen burning, the rapid proton capture (rp) process which would take place on a neutron star surface in X-ray burst is discussed together with a new X-ray observation that suggests the rp-process termination at around $\mathrm{A}=100$. The observation of the afterglow of the binary neutron star merger appears to be the kilo-nova predicted in the last decade in nuclear astrophysics, and to be the great success of the field. However, the detailed study of the kilo-nova by the r-process should be a great challenge for full understanding heavy element synthesis and the neutron star merger. Nuclear physics problems are discussed for the kilonova.
\end{abstract}

\section{Explosive nuclear burning on neutron stars}

The neutron stars (NS) seem quite interesting, and active objects in the universe. They were before thought to be quiet, dead objects at the end of stellar evolution. However, if binary stellar system involves a neutron star or two neutron stars, they revive and play a very active role for stellar evolution as well as for chemical evolution in the universe [1]. In addition, the property of neutron stars is of great interest for nuclear physics as they are almost like a giant atomic nuclei made of hadrons, but the real property is poorly known. This is a keen subject for nuclear physics to learn the equation of state of nuclear matter.

A type I X-ray burst occurs in a binary system of an NS and a main sequence star [2,3], whereas a kilo-nova occurs [4] in a binary system of two neutron stars (NS-NS), whose gravitational wave was observed for the first time in August, 2017 [5].

The site of the r-process has been long one of the major quests for nuclear astrophysics. The core-collapse supernovae were considered for many years for the most possible site for the r-process, but this possibility has met in the past decade a serious difficulty in producing the intense neutron density needed for the full r-process after including most updated

\footnotetext{
*Corresponding author: kubono@riken.jp
} 
physics like the neutrino process. Instead a NS-NS merger has been discussed extensively as a possible r-process site together with a suggestion of a kilo-nova (or macro-nova in other word) observation right after merging [4]. Here, the main energy source of the kilonova comes from the nuclear decays of the r-process nuclides. A successful gravitational wave detection from a NS-NS merger (GW170817) was reported together with an afterglow which lasted nearly 100 days. This was exactly what was predicted as the kilonova in nuclear astrophysics before the observation. And thus, this remarkable observation and identification of the r-process site is partly a triumph of nuclear astrophysics.

Neutron stars are also known to have a site that produces abundant neutron-deficient, radioactive nuclides explosively. The type I X-ray bursts, a thermonuclear burning on a surface of the neutron star in a binary system, have been a long standing subject of study in nuclear astrophysics in terms of the energy and elemental production as well as the characteristic X-ray curve observations.

The nuclear physics problems in these two sites involve almost all the domain of the N$\mathrm{Z}$ plane of nuclides, and the research efforts for them are discussed in the following sections.

\section{Waiting points at intermediate mass region in the rp-process}

The rapid proton-capture process (rp-process) is considered to drive the type I X-ray bursts on the surface of the neutron star in a binary system. The accreting hydrogen gas from the accompanying star makes a thermonuclear burning, which would produce nuclides up to mass 100 region [2,3]. Recent report of an X-ray burst observation suggests that the rp-process may run up to $A=100$ region [6].

However, the nucleosynthetic flow path and the nuclide productions are largely uncertain because nuclear physics of the nuclei on the possible pathway beyond ${ }^{56} \mathrm{Ni}$, especially at around the possible waiting points are still not known well yet [7]. Here, I will discuss the possible waiting point at ${ }^{72} \mathrm{Kr}$ [8], as an interesting and serious suggestion was obtained during the EURICA campaign in RIKEN.

At higher-temperature and high-density site of hydrogen, the 2p-process might proceed to make a nucleus of $A+2 p$ bypassing the beta decay of the waiting point nucleus $A$. For instance, at the possible ${ }^{72} \mathrm{Kr}$ waiting point, the beta decay of ${ }^{72} \mathrm{Kr}$ would compete with the $2 \mathrm{p}$-process. If the $2 \mathrm{p}$-process is very slow, the rp-process flow has to wait for the beta decay of $\mathrm{T}_{1 / 2}=17.1 \mathrm{sec}$. Since ordinary type I X-ray burst duration times are 20-30 sec, a large portion of the rp-process flow will stall at this point. To this problem, one needs to know the mass of the surrounding nuclei, especially of ${ }^{73} \mathrm{Rb}$.

Recently, an interesting experimental result was obtained that suggests a rough mass value for ${ }^{73} \mathrm{Rb}$ in a EURICA campaign experiment at RIKEN-RIBF [9]. Many neutron-deficient $\mathrm{Rb}$ isotopes were produced by fragmentation of ${ }^{124} \mathrm{Xe}$ at $345 \mathrm{AMeV}$, but no event of ${ }^{73} \mathrm{Rb}$ was observed although one expected a certain yield from the systematics in this mass region, whereas we saw roughly the expected yield for ${ }^{72} \mathrm{Rb}$. Since the EURICA setup was set at F11 which locates more than $100 \mathrm{~m}$ away from the production target, ${ }^{73} \mathrm{Rb}$ is considered to have decayed out during the flight. The estimated half-life is less than $60 \mathrm{~ns}$. It is considered to be a proton decay of the ${ }^{73} \mathrm{Rb}$ ground state. The decay energy is estimated to be more than $600 \mathrm{keV}$ by considering the single particle state in this mass region in a deformed potential. If the ${ }^{73} \mathrm{Rb}$ is unbound by more than $600 \mathrm{keV}$, the 2 p-process would be very much retarded, and thus this waiting point seems quite serious for the rp-process. The direct mass measurement of ${ }^{73} \mathrm{Rb}$ is of great interest, although it is not so easy to measure masses of such short 
lived nuclei. Together, one needs to study the nuclear structure near and above the proton threshold in the relevant nuclei in the vicinity.

\section{Termination process of the rp-process}

\author{
Haettner et al. [10] had \\ reported precision mass
} measurements of the nuclei near the rp-process path at $A=81-87$, and claimed that the rp-process would have a possible $\mathrm{ZrNb}$ cycle at this mass region, and thus the rp-process flow would be seriously influenced to go to heavier mass region at a certain temperature. However, this conclusion was found to be very suspicious because their analysis included some other masses not measured [10].

Recently, the masses of ${ }^{82} \mathrm{Zr}$ and ${ }^{84} \mathrm{Nb}$ were measured with high precision for the first time, and those of ${ }^{79} \mathrm{Y},{ }^{81} \mathrm{Zr}$, and ${ }^{83} \mathrm{Nb}$ were remeasured at the CSRe storage ring in Lanzhou, China. Figure 1 shows the alpha separation energies derived based on the new masses, indicating the $\mathrm{ZyNb}$ cycle seems disappearing [11]. Here, the high precision mass

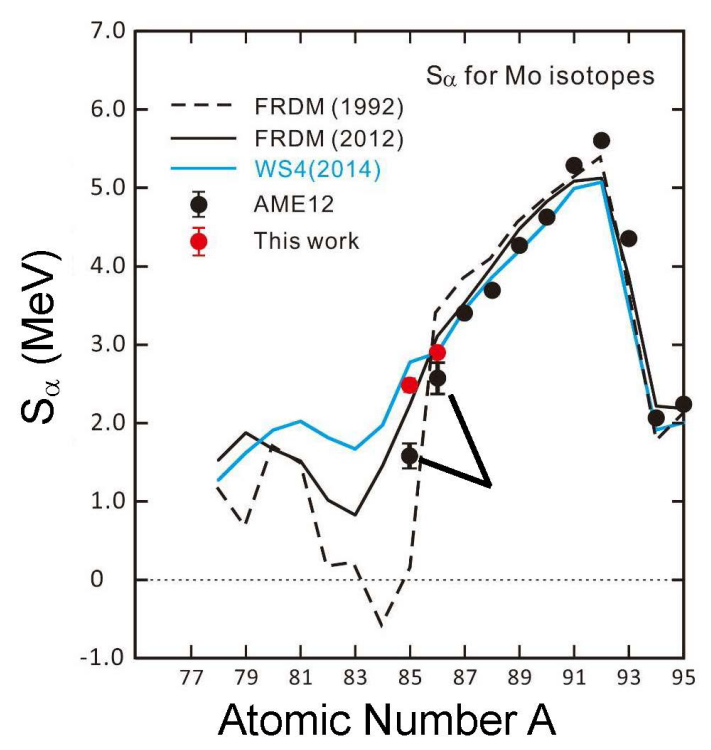

Fig. 1. The alpha separation energies were calculated based on the new masses determined at Lanzhou. The new data shows a smooth decrease suggesting the $\mathrm{ZrRb}$ cycle[10] would be less effective. [11]. measurement was made by the isochronous mass spectrometry, where the stored ions were set to the isochronous condition $\gamma=\gamma_{t}$ with $\gamma_{t}$ the transition energy of the ring [12]. Then, the precise $\mathrm{m} / \mathrm{q}$ value were extracted using reference particles whose masses are known with high precisions.

The new mass data have a considerable impact also to the p-nuclei production in this mass region, especially for production of ${ }^{84} \mathrm{Sr}$ in the vp-process. See ref. [11].

It should be of great interest if one can identify the elemental abundances produced in type I X-ray burst by astronomical observation.

\section{Experimental progress for the r-process nuclei}

Masses and half-lives have a decisive role in determining the pathway of the $r$ -

Fig. 2. The neutron decay path of ${ }^{86} \mathrm{Ge}$ determined in the BRIKEN Project. See ref. [15].

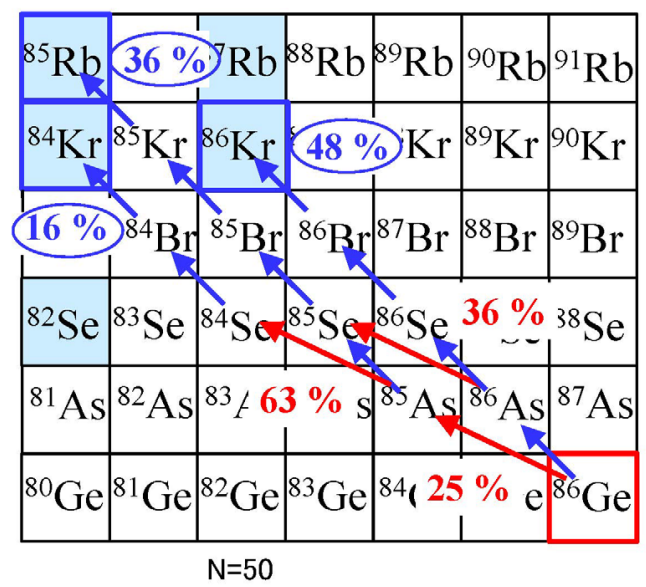


process under explosive nuclear burning. A systematic measurement of the half-lives was made for the very neutron-rich nuclei in the EURICA project $[13,14]$. The obtained halflives at $\mathrm{A}=100-175$ systematically deviate from any theoretical model predictions. Apparently, the r-process abundances were significantly changed by the new measurements of the half-lives. The project is extended to the BRIKEN campaign to measure the neutron emission probability Pn for the decay of very neutron-rich nuclei near the r-process path. The setup of BRIKEN will be found in ref. [15]. One of the first results [15] is illustrated in Fig. 2, where the decay of ${ }^{86} \mathrm{Ge}$ is illustrated. The decay paths are quite complicated, and change the final abundance quite significantly. The new decay paths reduce the ${ }^{86} \mathrm{Kr}$ production by $52 \%$, and instead the remaining $36 \%$ goes to ${ }^{85} \mathrm{Rb}$, and $16 \%$ to ${ }^{84} \mathrm{Kr}$. This example demonstrates how important the decay study of the r-process nuclei is.

\section{Experimental challenge for the kilo-novae}

The afterglow in the NS-NS merger, called kilo-nova or macro-nova, is the first evidence of the r-process site occurring in a stellar event, although a direct identification of each element synthesized was not made uniquely. The afterglow, measured by many telescopes in the world, had a specific light curve extending to a several tens of days after the merging. See Fig. 3. Wanajo[16] suggested that the shoulder at around 6-9 days in the luminosity curve could be explained by ${ }^{66} \mathrm{Ni}$ and ${ }^{72} \mathrm{Zn}$. More interesting is the extended curve beyond 20 days, which most probably come from the alpha and fission decays of actinide nuclei. Further detailed observation as well as nuclear physics study would be of great interest because neutron-rich actinides are still very poorly known yet. This study should be also relevant to the astronomical observations of $\mathrm{U}$ and $\mathrm{Th}$, and the fission recycling for the r-process.

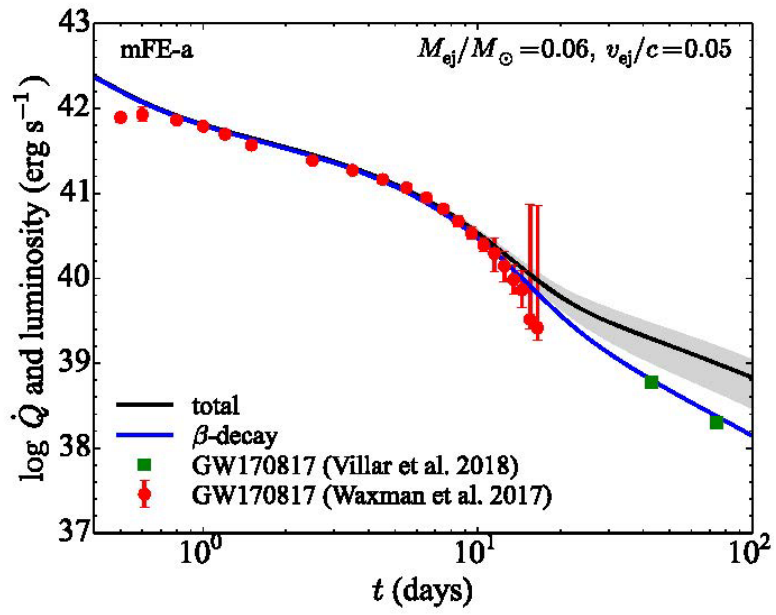

Fig. 3. The observed afterglow of the NS-NS merger of GW170817, and the lines are the analysis by Wanajo [16].

\section{Summary}

Neutron stars are quite active and interesting objects for nuclear astrophysics. The two major nucleosynthetic scenarios, the r-process and the rp-process would take place in a binary system that includes a neutron star or two neutron stars.

The detailed analysis of the kilo-nova observed recently was limited due to lack of nuclear data as well as the astronomical observation, whose efforts for the next NS-NS merger or NS-black hole merger will be improved including the KAGRA observatory [17] in Japan soon. Further detailed observations are of great interest in terms of chemical evolution as well as nuclear EOS, which will help to clarify the property of neutron stars. 
Experimental studies on these two nucleosynthetic scenarios were discussed here. Most nuclei on the possible rp-process are accessible in the laboratories and would be fully investigated in the coming years. However, most of the nuclei along the r-process are very difficult to approach yet. Only some nuclei around the $2^{\text {nd }}$ peak region are possible for the moment. Much more efforts are demanded in nuclear physics to approach to the r-process nuclei in the laboratories. New facilities coming up like FRIB, FAIR, HIAF and RAON as well as the upgraded RIBF will provide better chance for the study of the r-process in the laboratories.

\section{References}

1. Proceedings of the $14^{\text {th }}$ International Symposium on Nuclei in the Cosmos NIC2016, JPS Conference Proceedings No. 14 (2017) ed. S. Kubono, T. Kajino, S. Nishimura, T. Isobe, S. Nagataki, T. Shima, and Y. Takeda

Proceedings of the $15^{\text {th }}$ International Symposium on Nuclei in the Cosmos NIC2018, Gran Sasso, 2018, to be published

2. R. K.Wallace and S. E.Woosley, Astrophys. J. Suppl. Ser. 45, 389 (1981)

3. H. Schatz, et al., Phys. Rev. Lett. 86, 3471 (2001)

4. L.-X. Li and B. Pacznsky, Astrophys. J. Lett. 509, L59 (1998)

5. B.P. Abbott, et al., Phys. Rev. Lett. 119, 161101 (2017)

6. M. Kubota, et al., PASJ, in press

7. R.H. Cyburt, et al., Atrophys. J. 830, 55 (2016)

8. S. Kubono, $9^{\text {th }}$ European Summer School on Experimental Nuclear Astrophysics, EPJ Web Conferences 184, 01010 (2018)

9. H. Suzuki, et al., Phys. Rev. Lett. 119, 192503 (2017)

10. E. Haettner, et al., Phys. Rev. Lett. 106, 122501 (2011)

11. Y.M. Xing, et al., Phys. Lett. B 781, 358 (2018)

12. M. Hausmann, et al., Nucl. Instr. Meth. A 446, 569 (2000)

13. S. Nishimura, et al., Phys. Rev. Lett. 106, 052502 (2011)

14. J. Wu, et al., Phys. Rev. Lett. 118, 072701 (2017)

15. A. Tolosa-Delgadoa, et al., Nucl. Instr. Meth. A 925, 133 (2019)

16. S. Wanajo, Astrophys. J. 868, 65 (2018)

17. KAGRA Observatory, the University of Tokyo 